\title{
Unionsrechtliche Rechtskraftdurchbrechung? - Zu den Grenzen der mitgliedstaatlichen Verfahrensautonomie auf dem Gebiet der Rechtskraft nach der Rechtsprechung des EuGH
}

\author{
Swantje Wagner*
}

Inhalt
A. Einführung
B. Die Funktion der Rechtskraft
C. Die Verfahrensautonomie der Mitgliedstaaten und ihre Grenzen
D. Die Lösung durch den EuGH
I. Die unionsrechtliche Staatshaftung für judikatives Unrecht
II. Die Bestandskraftdurchbrechung gerichtlich bestätigter Verwaltungsentscheidungen
III. Kollisionen im Beihilfen- und Steuerrecht
E. Die Auswirkungen auf das Rechtskraftprinzip der Mitgliedstaaten

\section{A. Einführung}

Ein gerichtliches Verfahren erfüllt eine streitschlichtende und eine friedensstiftende Funktion und entspricht so einer wirksamen und ausgewogenen Rechtsschutzgewährung durch die Gerichte. ${ }^{1}$ Von zentraler Bedeutung ist dabei die Endgültigkeit der Streitentscheidung, denn sie schafft Rechtssicherheit. Dies wird insbesondere durch das Institut der Rechtskraft realisiert, welches bewirkt, dass Gerichtsentscheidungen nach Ausschöpfung des Rechtswegs oder nach Ablauf der entsprechenden Rechtsmittelfristen unanfechtbar werden und die Verfahrensbeteiligten binden. Die Rechtskraft ist als allgemeiner Prozessrechtsgrundsatz in jeder modernen Rechtsordnung anerkannt, auch im Unionsrecht. ${ }^{2}$ Die Umsetzung des Unionsrechts erfolgt durch die Mitgliedstaaten, vor allem durch die nationalen Gerichte. Die nationalen Rechtskraftregelungen sichern daher auch die Verbindlichkeit und Dauer-

* Der Beitrag basiert auf einer Magisterarbeit der Autorin zum Erwerb des M.L.E. im Ergänzungsstudiengang „Rechtsintegration in Europa“ an der Georg-August-Universität Göttingen. Für die Betreuung der Magisterarbeit und die Unterstützung bei der Anfertigung dieses Aufsatzes sei Prof. Dr. José Martínez herzlich gedankt.

1 Vgl. BFH, Beschl. v. 18.8.2005, VI R 123/94, Rdnr. 19; Schilken, Gerichtsverfassungsrecht, 4. Aufl. 2007, S. 41 ff.; Schachtschneider, Prinzipien des Rechtsstaates, 2006, S. 143.

2 Germelmann, Die Rechtskraft von Gerichtsentscheidungen in der Europäischen Union, 2009, S. 1. 
haftigkeit der unionsrechtlichen Vorgaben. Das Rechtskraftprinzip ist folglich ein wichtiger Baustein für das Unionsrecht, weshalb der Gerichtshof seine Bedeutung fortwährend herausgestellt hat. ${ }^{3}$ Beim Vollzug des Unionsrechts entstehen durch das Aufeinandertreffen der nationalen Rechtsordnungen und der Unionsrechtsordnung besondere Herausforderungen: Indem die Mitgliedstaaten die Modalitäten der Rechtskraft ihrer gerichtlichen Entscheidungen autonom festlegen, können sie auch die Korrektur unionsrechtswidriger Judikate behindern. Fraglich ist, inwieweit das Gebot des effet utile der Rechtskraft mitgliedstaatlicher Gerichtsentscheidungen Grenzen setzt, wenn diese sich als unionsrechtswidrig herausstellen. Nachstehend soll daher der Einfluss des Unionsrechts auf das Rechtskraftprinzip der Mitgliedstaaten untersucht werden. Dazu sind zunächst die Situationen zu analysieren, in denen das Unionsrecht eine Abänderung des rechtskräftigen Urteils verlangt oder zugunsten der Rechtssicherheit und anderer Prinzipien darauf verzichtet. Im Fall einer unionsrechtlich geforderten Rechtskraftdurchbrechung müsste das nationale Verfahrensrecht entsprechende Möglichkeiten bieten, den unionsrechtlichen Vorgaben nachzukommen. Dies soll exemplarisch anhand des deutschen und englischen Rechts untersucht werden. Diese Rechtsordnungen bieten sich an, da das deutsche Recht als eine der großen Gründungsrechtsordnungen der Europäischen Wirtschaftsgemeinschaft die Rechtsprechung des EuGH maßgeblich beeinflusst hat und das zum angloamerikanischen Rechtskreis gehörende englische Recht erheblich durch Gerichtsentscheidungen geprägt wird und so dem deutschen Rechtssystem entgegengestellt werden kann.

\section{B. Die Funktion der Rechtskraft}

Die Rechtskraft sichert die subjektiven Rechtspositionen des von der Gerichtsentscheidung Betroffenen und garantiert zugleich die Wirksamkeit der Entscheidungen des rechtsprechenden Organs. Damit gewährleistet sie Rechtsfrieden, die Beständigkeit rechtlicher Beziehungen und eine geordnete Rechtspflege. ${ }^{4}$ Die hinter der Rechtskraft stehende Idee der Schaffung von Rechtssicherheit kann auf die römisch-rechtliche Maxime res judicata pro veritate accipitur bzw. habetur zurückgeführt werden: Das Urteil wird als bestehende Wahrheit angenommen bzw. für wahr gehalten. Dies bedeutet, dass ein rechtskräftiges Urteil nicht die Wahrheit bildet, sondern als wahr gilt, weil es nicht mehr in Frage gestellt werden kann. ${ }^{5}$ Dass inhaltlich falsche Entscheidungen unanfechtbar werden, ist das immanente Risiko der Rechtskraft. ${ }^{6}$ Die Rechtssicherheit und mit ihr die Rechtskraft stehen grundsätzlich keinesfalls im Gegensatz zur Gesetzmäßigkeit, da sie zugleich selbst Ele-

3 Vgl. EuGH, Rs. C-224/01, Köbler, Slg. 2003, I-10239, Rdnr. 38; EuGH, Rs. C-234/04, Kapferer, Slg. 2006, I-2585, Rdnr. 20; EuGH, Rs. C-2/08, Olimpiclub, Slg. 2009, I-7501, Rdnr. 22; EuGH, Rs. C-40/08, Asturcom, Slg. 2009, I-9579, Rdnr. 36; EuGH, Rs. C-507/08, Kommission/Slowakische Republik, Slg. 2010, I-13489, Rdnr. 59.

4 EuGH, Rs. C-2/08, Olimpiclub, Slg. 2009, I-7501, Rdnr. 22.

5 Germelmann, (Fn. 2), S. 343; von Savigny, System des heutigen Römischen Rechts, Bd. VI, 1847, $\S 280$, S. 261 ff.; Gaul, in: Festschrift Flume, Bd. I, 1978, S. 455.

6 Reiling, Streitgegenstand und Einrede der „res iudicata“ in Direktklageverfahren vor den Gemeinschaftsgerichten, EuZW 2002, S. 136. 
mente der Gerechtigkeit sind. ${ }^{7}$ Die jeder Rechtsordnung eigenen Gesetzmäßigkeitszwecke werden durch die Beständigkeit der Rechtsverhältnisse verwirklicht, die unter ihrem Schutz entstehen. Hauptziel jeder Rechtsordnung ist aber nicht die Gerechtigkeit, sondern die Rechtsbeziehungen zu ordnen, was durch die Unangreifbarkeit bestandskräftiger Rechtsakte geschieht. ${ }^{8}$ Ein materiell unrichtiges oder ungerechtes Urteil muss angesichts der sonst entstehenden Rechtsunsicherheit in Kauf genommen werden. ${ }^{9}$

Allerdings kennt das Recht keine absolut wirkende Veränderungssperre, weswegen das rechtskräftige Urteil überprüft und gegebenenfalls revidiert werden kann. ${ }^{10}$ Das Verhältnis zwischen dem Streben nach Gerechtigkeit und der Notwendigkeit, Rechtssicherheit zu gewährleisten, ist in den verschiedenen europäischen Rechtskulturen unterschiedlich ausgestaltet. Daher divergiert die prozessuale Rechtssicherheit in den Rechtsordnungen erheblich in ihrer konkreten Zielsetzung, in ihrer dogmatischen Begründung im materiellen Recht oder Prozessrecht und in ihrer Bedeutung der objektiv- oder subjektiv-rechtlichen Komponente der Rechtskraft.

Gerichtliche Entscheidungen können über die Entscheidung des Einzelfalls hinaus durch die Klärung zweifelhafter und umstrittener Rechtsfragen generelle Bedeutung erlangen, wobei diese Präjudizwirkung keinesfalls mit der Rechtskraft gleichgestellt werden darf. ${ }^{11}$ Voraussetzung für die Rechtsfortbildung ist jedoch, dass der Inhalt der Entscheidung maßgeblich und rechtsbeständig bleibt. ${ }^{12}$ Der Rechtskraft kommt somit eine wichtige Funktion bei der Fortbildung des Rechts und für Rechtssysteme zu, die ausschlaggebend von Gerichtsentscheidungen geprägt werden. Dies betrifft nicht nur das common law und die Rechtspraxis der kontinentalen Rechtsordnungen, sondern auch das Unionsrecht, denn der EuGH hat mit seiner richterlichen Rechtsfortbildung die Entwicklung des Unionsrechts erheblich beeinflusst. ${ }^{13}$

7 Schlussanträge GA Colomer zu EuGH, Rs. C-310/97 P, Kommission/AssiDomän Kraft Products u.a., Slg. 1999, I-5363, 5380 f.; Schachtschneider, Neubescheidung nach Rechtskraft im Sozialversicherungsrecht und im allgemeinen Verwaltungsrecht, VerwArch 63 (1972), S. 306.

8 Schlussanträge GA Colomer zu EuGH, Rs. C-310/97 P, Kommission/AssiDomän Kraft Products u.a., Slg. 1999, I-5363, 5380 f., wobei sich GA Colomer explizit auf Goethes Rechtsauffassung bezieht, wonach Unrecht besser sei, als eine Welt ohne Gesetz, aber in ihrer Absolutheit zurückweist. Die Aufgabe des Rechts als Ordnungsfaktor des sozialen und staatlichen Lebens wird von der Radbruch'schen Formel nicht in Frage gestellt, sondern lediglich mit einem Vorbehalt versehen, was das Recht aber nur mittels Beständigkeit und Dauerhaftigkeit erfüllen kann, siehe von Arnauld, Rechtssicherheit, 2006, S. 637; Maurer, in: Isensee/Kirchhof (Hrsg.), HStR, Bd. IV, 2006, § 79, Rdnr. 1; BVerfGE 35, 41 (53 f.).

9 Reiling, (Fn. 6), S. 136; Sobota, Das Prinzip Rechtsstaat, 1997, S. 179 f.

10 Vgl. von Arnauld, (Fn. 8), S. 276; Maurer, (Fn. 8), § 79, Rdnr. 134.

11 Ibid., Rdnr. 135; Lames, Rechtsfortbildung als Prozeßzweck, 1993, S. 32 f.

12 Kremer, Gemeinschaftsrechtliche Grenzen der Rechtskraft, EuR 2007, S. 471.

13 Zur Praxis auf dem Kontinent: Zweigert/Kötz, Einführung in die Rechtsvergleichung, 3. Aufl. 1996, S. 256 f.; Kremer, (Fn. 12), S. 471 f. m.w.N.; zum Unionsrecht: Herdegen, Europarecht, 14. Aufl. 2012, S. 142, Rdnr. 98; Pechstein, EU-Prozessrecht, 4. Aufl. 2011, S. 6, Rdnr. 9. 


\section{Die Verfahrensautonomie der Mitgliedstaaten und ihre Grenzen}

Artikel 5 Abs. 2 EUV bestimmt, dass die Union nach dem Prinzip der begrenzten Einzelermächtigung nur innerhalb der Grenzen der ihr übertragenen Zuständigkeiten tätig wird. Eine normative Kompetenz im Bereich des Verfahrensrechts besitzt die Union nicht. ${ }^{14}$ Soweit keine unionsrechtlichen Regelungen vorhanden sind, gehen die Mitgliedstaaten bei der Durchführung des Unionsrechts nach den formellen und materiellen Bestimmungen ihres nationalen Rechts vor (sogenannte Soweit-Formel). ${ }^{15}$ Die selbständige Wahl der Mittel zur Gewährleistung des materiellen EU-Rechts wird auch als Verfahrensautonomie oder national procedural autonomy bezeichnet. ${ }^{16}$ Artikel 4 Abs. 3 EUV verpflichtet die Mitgliedstaaten zu einem vollständigen, einheitlichen und effektiven Vollzug des Unionsrechts. ${ }^{17}$ Dies kann auch als „Nebeneffekt aus den unionsrechtlichen Vorschriften zum materiellen Recht" ${ }^{* 18}$ bezeichnet werden. Daraus entsteht in Bezug auf die Rechtskraft folgendes Spannungsverhältnis: Ist eine nationale rechtskräftige Gerichtsentscheidung unionsrechtswidrig, entsteht ein Konflikt zwischen den Änderungsinteressen zugunsten der realen Geltung des Unionsrechts und den Interessen an der Gültigkeit der mitgliedstaatlichen Rechtskraftregelung. Zur Lösung dieser indirekten Kollision kann jedenfalls das Prinzip des Vorrangs nicht herangezogen werden, da die Union in diesem Bereich keine normative Kompetenz besitzt. Der EuGH hat die externe Grenze der Verfahrensautonomie folgendermaßen bestimmt: Es muss das Gebot berücksichtigt werden, keine Schlechterbehandlung von unionsrechtlichen Fällen im Vergleich zu gleichartigen rein nationalen Fällen zu ermöglichen (Äquivalenzgebot) und die Verwirklichung der Regelung der Union nicht praktisch unmöglich zu machen oder übermäßig zu erschweren (Effektivitätsgebot). ${ }^{19}$ Grundsätzlich wird daher die Kompetenz der Mitgliedstaaten im Konfliktfall anerkannt, die Anwendung der nationalen Verfahrensvorschriften aber an der Effektivität des

14 Galetta, Begriff und Grenzen der Verfahrensautonomie der Mitgliedstaaten der Europäischen Union, EuR-Beiheft 1/2012, S. 38, mit dem Hinweis, dass Art. 115 und 114 AEUV z.B. im Fall der Richtlinien zum Nachprüfverfahren bzgl. der Vergabe öffentlicher Aufträge eine Ausnahme darstellen.

15 EuGH, Rs. 94/71, Schlüter \& Maack, Slg. 1972, 307, Rdnr. 10; EuGH, verb. Rs. 205-215/82, Deutsche Milchkontor u.a., Slg. 1983, 2633, Rdnr. 17; König, Der Äquivalenz- und Effektivitätsgrundsatz in der Rechtsprechung des Europäischen Gerichtshofs, 2011, S. 17.

16 Vgl. von Danwitz, Europäisches Verwaltungsrecht, 2008, S. 302; König, (Fn. 15), S. 29; Kremer (Fn. 12), S. 473; Craig/de Búrca, EU Law, 5. Aufl. 2011, S. 231. Diese Begriffe werden teilweise mit der Begründung abgelehnt, sie seien wenig präzise und missverständlich, denn sie suggerierten eine Immunität gegen unionsrechtliche Vorgaben, die es so nicht gebe, da diese jedenfalls zu beachten seien. Stattdessen sei der Begriff Verfahrenskompetenz bzw. procedural competence vorzuziehen, siehe Bieber/Epiney/Haag, Die Europäische Union, 9. Aufl. 2011, S. 226, Fn. 29; van Gerven, Of Rights, Remedies and Procedures, CMLR 2000, S. 502. Inhaltlich unterscheiden sich diese Ansichten zur Begriffsverwendung nicht.

$17 \mathrm{Kahl}$, in: Calliess/Ruffert (Hrsg.), EUV/AEUV, 4. Aufl. 2011, Art. 4 EUV, Rdnr. 62.

18 Galetta, in: Schwarze (Hrsg.), Das Verhältnis von nationalem Recht und Europarecht im Wandel der Zeit, 2012, S. $180 \mathrm{f}$.

19 EuGH, Rs. 33/76, Rewe, Slg. 1976, 1989, Rdnr. 5; EuGH, verb. Rs. C-430/93 und C-431/93, van Schijndel, Slg. 1995, I-4705, Rdnr. 17 ff.; Galetta, Procedural Autonomy of EU Member States: Paradise Lost?, 2010, S. 16 ff.; Haratsch/Koenig/Pechstein, Europarecht, 8. Aufl. 2012, S. 264, Rdnr. 593. 
Unionsrechts gemessen. ${ }^{20}$ Einwirkungen auf das mitgliedstaatliche Recht sind rechtfertigungsbedürftig und nur insoweit zulässig, wie sie zur Gewährleistung der Wirksamkeit des Unionsrechts erforderlich sind. ${ }^{21}$ Fraglich ist, in welchen Konstellationen ein europarechtskonformer Zustand wiederhergestellt und die Rechtskraft durchbrochen werden muss. Inwieweit erweist sich also die Verfahrensautonomie in der Rechtsprechung des EuGH als Mythos oder Realität? Dabei stellen Vertragsverletzungs- und Vorabentscheidungsverfahren die häufigsten Überprüfungsmöglichkeiten des nationalen Verfahrensrechts für den EuGH dar.

\section{Die Lösung durch den EuGH}

\section{Die unionsrechtliche Staatshaftung für judikatives Unrecht}

Mit der Entscheidung in der Rechtssache Köbler vollendete der Gerichtshof sein durch richterliche Rechtsfortbildung entwickeltes Haftungssystem für die Mitgliedstaaten, indem er einen grundsätzlichen Haftungsanspruch für unionsrechtswidrige Fehlentscheidungen letztinstanzlicher Gerichte bejahte. ${ }^{22}$ Im Ausgangsverfahren hatte der österreichische Universitätsprofessor Köbler die Republik Österreich auf Ersatz des Schadens verklagt, der ihm dadurch entstanden sei, dass der Verwaltungsgerichtshof ein Vorabentscheidungsersuchen nicht aufrecht erhalten und Unionsrecht falsch angewendet habe. Obgleich im vorliegenden Fall kein Haftungsanspruch bestand, stellte der EuGH klar, dass die Mitgliedstaaten unabhängig davon haften, ob der schadensverursachende Unionsrechtsverstoß der Legislative, der Exekutive oder der Judikative zuzurechnen ist. ${ }^{23}$ Fraglich ist, ob dies als rechtfertigungsbedürftiger Eingriff in die Verfahrensautonomie der Mitgliedstaaten zu werten ist und die Wirkung der Rechtskraft nationaler Gerichtsentscheidungen dadurch beschränkt wird.

Einen Haftungsanspruch für Gerichtsentscheidungen hatten die Regierungen Frankreichs und des Vereinigten Königreichs in ihren Stellungnahmen zum Verfahren unter anderem deshalb abgelehnt, da dies dem Prinzip der Rechtssicherheit und Rechtskraft widerspräche: Die Anerkennung der Staathaftung für judikatives Unrecht führe zu einer erneuten Verhandlung der Streitfragen und würde die Prozessparteien in Unsicherheit bezüglich ihrer Rechtspositionen lassen. ${ }^{24}$ Diese Kritik ist von Teilen der Literatur bestätigt

20 König, (Fn. 15), S. 43; andere Ansicht Huthmacher, Der Vorrang des Gemeinschaftsrechts bei indirekten Kollisionen, 1985, S. 149 ff., der die Vorrangregel auch bei indirekten Kollisionen anwenden möchte, was zu denselben Ergebnissen führen kann, da auch diese Ansicht auf die Wirksamkeit des Unionsrechts ausgerichtet ist, jedoch gegen das primärrechtliche Kompetenzgefüge verstößt. Zudem geht der EuGH von der Anwendbarkeit der nationalen Rechtsvorschriften aus, siehe EuGH, Rs. 45/76, Comet, Slg. 1976, 2045, 2053; EuGH, Rs. C-255/00, Grundig Italiana, Slg. 2002, I-8003, 8026; von Danwitz, (Fn. 16), S. 155; König, (Fn. 15), S. 32 f.

21 Schlussanträge GA Jacobs zu EuGH, verb. Rs. C-430/93 und C-431/93, van Schijndel, Slg. 1995, I-4705, Nr. 31; von Danwitz, (Fn. 16), S. 311; König, (Fn. 15), S. 106 f.

22 Kluth, Die Haftung der Mitgliedstaaten für gemeinschaftsrechtswidrige höchstrichterliche Entscheidungen - Schlussstein im System der gemeinschaftsrechtlichen Staatshaftung, DVB1. 2004, S. 399.

23 EuGH, Rs. C-224/01, Köbler, Slg. 2003, I-10239, Rdnr. 32.

24 Ibid., Rdnrn. 23, 25. 
worden, denn im Folgeprozess müsse notwendigerweise zunächst die Fehlerhaftigkeit des Urteils festgestellt werden, um die finanziellen Auswirkungen anschließend korrigieren zu können. ${ }^{25}$ Doch der Gerichtshof sowie Generalanwalt Léger hielten das Rechtskraftprinzip mit einem Staatshaftungsanspruch für vereinbar. Der Haftungsprozess habe nicht denselben Gegenstand und nicht zwangsläufig dieselben Parteien wie das Verfahren, das zur rechtskräftigen Entscheidung geführt hat. Zudem verlange das Unionsrecht lediglich eine Entschädigung, aber keine Abänderung der schadensbegründenden Gerichtsentscheidung. ${ }^{26}$ Obgleich es zutrifft, dass mit dem Haftungsprozess ein anderes Klageziel als im Ausgangsverfahren verfolgt wird und damit formal verschiedene Streitgegenstände vorliegen, wird die Lösung des EuGH der Bedeutung der Rechtskraft nicht gerecht. Die Rückabwicklung im Wege der Staatshaftung führt dazu, dass die Richtigkeit der rechtskräftigen Entscheidung erneut zur Debatte steht und damit die Befriedigungswirkung aufgehoben wird. Doch der EuGH prüft die Aspekte der Rechtskraft nur auf der Grundsatzebene des „ob“ der Staatshaftung, weshalb seine Begründung nicht überzeugt. ${ }^{27}$

Insgesamt ist die Haftung des Staates für EU-Rechtsverstöße nationaler Höchstgerichte zu begrüßen, denn dadurch werden die dem Einzelnen aufgrund unionsrechtlicher Bestimmungen zustehenden Rechte effektiv geschützt. ${ }^{28}$ Bei der damit verbundenen objektiven Wirksamkeit des Unionsrechts muss allerdings beachtet werden, dass der Rechtsprechung gegenüber der Legislative und der Exekutive eine besondere Funktion zukommt, die für die staatliche Gemeinschaft von elementarem Interesse ist: Als dritte Staatsgewalt kontrolliert sie unabhängig und unparteilich die Ausübung öffentlicher Gewalt gegenüber dem Bürger. ${ }^{29}$ Die richterliche Unabhängigkeit darf daher durch den Haftungsanspruch nicht gefährdet werden, wie es etwa das Vereinigte Königreich in seiner Stellungnahme zum Verfahren befürchtete. ${ }^{30}$ Dieses Argument wies der EuGH jedoch zurück, da es nicht um die persönliche Haftung des Richters gehe, sondern um die des Staates. ${ }^{31}$ In erster Linie wird daher eine staatliche Haftung für Fehlentscheidungen unionsrechtlich gefordert. Dies kann jedoch dann die Entscheidungsfreiheit und -freude des Richters gefährden, wenn die

25 Bereits Wegener, Staatshaftung für die Verletzung von Gemeinschaftsrecht durch nationale Gerichte, EuR 2002, S. 794, Fn. 49; Radermacher, Gemeinschaftsrechtliche Staatshaftung für höchstrichterliche Entscheidungen, NVwZ 2004, S. 1418; Kremer, (Fn. 12), S. 475; Komárek, Federal elements in the Community judicial system: Building coherence in the Community legal order; CMLR 2005, S. 28; Wattel, Köbler, CILFIT and Welthgrove: We can't go on meeting like this, CMLR 2004, S. 177; Nassimpian, And we keep on meeting: (de)fragmenting state liability, European Law Review 2007, S. 835 f.

26 EuGH, Rs. C-244/01, Köbler, Slg. 2003, I-10239, Rdnr. 38; dazu Schlussanträge GA Léger, Nr. 101.

27 Radermacher, (Fn. 25), S. $1417 \mathrm{f}$.

28 EuGH, Rs. C-224/01, Köbler, Slg. 2003, I-10239, Rdnr. 33; Groussot/Minssen, Res judicata in the Court of Justice case-law: balancing legal certainty with legality?, European Constitutional Law Review 2007, S. 395.

29 Hillgruber, in: Maunz/Dürig (Hrsg.), GG, Stand 12/2007, Art. 92, Rdnr. 8 ff.

30 EuGH, Rs. C-224/01, Köbler, Slg. 2003, I-10239, Rdnr. 26; ebenso Lord Salmon, in: Sutcliff v. Thackrah [1974] 1 All E.R. 859 (881).

31 EuGH, Rs. C-224/01, Köbler, Slg. 2003, I-10239, Rdnr. 41 f.; Wegener, (Fn. 25), S. 793; Zenner, Die Haftung der EG-Mitgliedstaaten für die Anwendung europarechtswidriger Rechtsnormen, 1995, S. 239 f. 
Grenzen für einen Regressanspruch gegen ihn nicht hinreichend eng gezogen sind. ${ }^{32}$ Es bleibt bisher den Mitgliedstaaten überlassen, einen Rückgriff auf den fehlurteilenden Richter so auszugestalten, dass seine Unabhängigkeit nicht gefährdet wird. Der Haftungsanspruch selbst gefährdet sie nicht. Der Gerichtshof könnte mit Rücksicht auf die Verfahrensautonomie der Mitgliedstaaten von Vorgaben für den Regress abgesehen haben, denn er überließ es auch ausdrücklich den nationalen Rechtsordnungen, die Zuständigkeit der Gerichte für künftige Haftungsprozesse zu regeln. ${ }^{33}$

Obgleich der EuGH die Rechtskraft und richterliche Unabhängigkeit nicht gefährdet sah, privilegiert er Gerichtsentscheidungen im unionsrechtlichen Haftungssystem. Ohne eine Haftungsbeschränkung für judikatives Unrecht müsste die Richtigkeit von (auch höchstrichterlichen) Entscheidungen anderer Gerichtsbarkeiten im Haftungsprozess überprüft werden, was den Instanzenzug und die Zuordnung unterschiedlicher Rechtsfragen zu unterschiedlichen Teilgerichtsbarkeiten relativieren würde. ${ }^{34}$ Zudem bestände die Gefahr, dass die Entscheidung im Haftungsprozess selbst wieder zum Gegenstand eines weiteren Staatshaftungsprozesses gemacht und ein unendlicher Rechtsstreit geführt wird. ${ }^{35}$ Dies würde einer funktionierenden Rechtspflege, der Rechtssicherheit und der Friedensfunktion der Rechtsprechung widersprechen, die für den zur Justizgewährleistung verpflichteten Rechtsstaat unverzichtbar sind. Dementsprechend berücksichtigt der EuGH ,die Besonderheit der richterlichen Funktion sowie die berechtigten Belange der Rechtssicher-

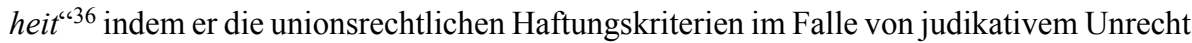
in der Rechtssache Köbler modifiziert hat.

Bisher wurde unter drei Voraussetzungen die mitgliedstaatliche Haftung ausgelöst: Die verletzte Norm muss bezwecken, dem Einzelnen Rechte zu verleihen, das Unionsrecht

32 Wegener, (Fehl-)Urteilsverantwortung und Richterspruchprivileg in der Haftung der Mitgliedstaaten für die Verletzung von Gemeinschaftsrecht, EuR 2004, S. 88; Grune, Staatshaftung bei Verstößen nationaler Gerichte gegen Europäisches Gemeinschaftsrecht, BayVBl. 2004, S. 676, die jedoch bezweifeln, dass durch einen Regress überhaupt die richterliche Unabhängigkeit gefährdet wird, da es keinen Grund gebe, den Richter im Gegensatz zu anderen Amtsträgern besser zu stellen.

33 EuGH, Rs. C-224/01, Köbler, Slg. 2003, I-10239, Rdnr. 45; zur Autonomie im Staatshaftungsprozess siehe Kischel, Gemeinschaftsrechtliche Staatshaftung zwischen Europarecht und nationaler Rechtsordnung, EuR 2005, S. $450 \mathrm{f}$.

34 Wegener, (Fn. 25), S. 794 f.; Grune, (Fn. 32), S. 677.

35 Ibid.; Wegener, (Fn. 25), S. $794 \mathrm{f}$.

36 EuGH, Rs. C-224/01, Köbler, Slg. 2003, I-10239, Rdnr. 53. Das Richterspruchprivileg begründet der EuGH nicht näher. Die Sicherung der Funktionsfähigkeit der Rechtsprechung ist aber neben der von ihm als ungefährdet angesehenen Rechtskraft das überzeugendste Argument. Auch lässt der EuGH die Mitgliedstaaten mit den Problemen (Instanzenzug, Gerichtsbarkeit, Rechtssicherheit) bei der Ausgestaltung des Haftungsprozesses allein, indem es ihre Sache sei, einen geeigneten Rechtsweg zu Verfügung zu stellen, siehe ibid., Rdnr. 45. Insbesondere die Bestimmung des zuständigen Gerichts stellt einige Mitgliedstaaten vor erhebliche Probleme, siehe Zenner, (Fn. 31), S. 229 ff.; insbesondere im englischen Recht siehe Giliker, English tort law and the challenge of Francovich liability: 20 years on, The Law Quaterly Review 2012, S. 563; Zuckerman, „Appeal“ to the High Court against House of Lords decisions on the interpretation of Community law - damages for judicial error, Civil Justice Quaterly 2004, S. 9 ff. Das Vereinigte Königreich hatte gegen den Haftungsanspruch gerade damit argumentiert, dass es ihn im englischen Recht nicht gebe, siehe EuGH, Rs. C-224/01, Köbler, Slg. 2003, I-10239, Rdnr. 24. 
muss hinreichend qualifiziert sein und ein unmittelbarer Kausalzusammenhang zwischen dem Verstoß und dem eingetretenen Schaden muss bestehen. ${ }^{37}$

Auf der Ebene der Qualifiziertheit des Unionsrechtsverstoßes fordert der EuGH für einen Haftungsanspruch aufgrund einer Gerichtsentscheidung einen offenkundigen Verstoß. ${ }^{38}$ Durch diese enge Voraussetzung verzichtet er zugunsten der Verfahrensautonomie auf eine maximale Durchsetzung des Unionsrechts im Sinne des Effektivitätsprinzips. ${ }^{39}$ Die zurückhaltende Tendenz des Gerichtshofs wird auch daran deutlich, dass Generalanwalt Léger unter Verwendung im Wesentlichen gleicher Kriterien zu einem offenkundigen Verstoß gelangte. ${ }^{40}$

Wann genau Offenkundigkeit vorliegt, ist bisher noch unklar geblieben. Die Verletzung der Vorlagepflicht des letztinstanzlichen Gerichts aus Art. 267 Abs. 3 AEUV hatte der EuGH zwar in diesem Zusammenhang geprüft, die Voraussetzung sei dadurch aber nicht zwingend erfüllt. ${ }^{41}$ Teilweise wird in der Literatur der Staatshaftungsprozess als eine Art „Wunderwaffe“42 zur Sanktionierung der Vorlagepflichtverletzung verstanden, was sonst nur mithilfe eines Vertragsverletzungsverfahrens möglich wäre. ${ }^{43}$ Diese Ansicht berücksichtigt jedoch nicht, dass mangels individualschützender Wirkung die alleinige Verletzung des Art. 267 Abs. 3 AEUV die erste Haftungsvoraussetzung nicht erfüllt. ${ }^{44}$ Die Vorlagepflichtverletzung ist aber bei der Beurteilung der Offenkundigkeit im Einzelfall zu berücksichtigen.

Dies ist in der Rechtssache Traghetti bestätigt worden: Ein italienisches Unternehmen verklagte die Republik Italien auf Schadensersatz wegen der vermeintlich falschen Auslegung der unionsrechtlichen Wettbewerbsbestimmungen durch ein letztinstanzliches Gericht und insbesondere dessen Nichtvorlage an den EuGH. ${ }^{45}$ Dieser sah auch in diesem Verfahren davon ab, die Vorlagepflichtverletzung als eigenständiges Haftungskriterium heranzuziehen. Die Gründe hierfür lassen sich erahnen: Die Auslegung und Anwendung

37 EuGH, verb. Rs. C-46/93 und C-48/93, Brasserie du pêcheur und Factortame, Slg. 1996, I-1029, Rdnr. 51.

38 EuGH, Rs. C-224/01, Köbler, Slg. 2003, I-10239, Rdnr. 53.

39 Gundel, Gemeinschaftsrechtliche Haftungsvorgaben für judikatives Unrecht - Konsequenzen für die Rechtskraft und das deutsche „Richterprivileg“ (§ 839 Abs. 2 BGB), EWS 2004, S. 15; Kremer, (Fn. 12), S. 476; Obwexer, Anmerkung, EuZW 2003, S. 728.

40 Schlussanträge GA Léger zu EuGH, Rs. C-224/01, Köbler, Slg. 2003, I-10239, Nr. 170; Wegener, (Fn. 32), S. 91.

41 EuGH, Rs. C-224/01, Köbler, Slg. 2003, I-10239, Rdnr. 123 f.; aber in seinen Schlussanträgen weißt GA Léger, Nr. 148 darauf hin, dass die Vorlagepflichtverletzung die Staatshaftung auslösen kann.

42 Hakenberg, Zur Staatshaftung von Gerichten bei Verletzung von europäischem Gemeinschaftsrecht, DRiZ 2004, S. 117.

43 Storr, Abschied vom Spruchrichterprivileg?, DÖV 2004, S. 548; Kenntner, Ein Dreizack für die offene Flanke: Die neue EuGH-Rechtsprechung zur judikativen Gemeinschaftsrechtsverletzung, EuZW 2005, S. 237.

44 Frenz/Götzkes, Staatshaftung für Gerichtsentscheidungen bei auslegungsbedürftigem Recht, EuR 2009, S. 629; auch Obwexer, (Fn. 39), S. 728, demzufolge die Möglichkeit bestände, dass der Einzelne die Verletzung seines unionsrechtlich geltenden Grundrechts auf Gerichtszugang geltend macht, doch würde die Vorlagepflichtverletzung auch hier in Verbindung mit einer anderen falsch ausgelegten oder angewandten Bestimmung des Unionsrechts stehen, zudem sei der Kausalzusammenhang schwer nachzuweisen.

45 EuGH, Rs. C-173/03, Traghetti/Italienische Republik, Slg. 2006, I-5177, Rdnrn. 32, 43. 
des Unionsrechts wird wesentlich durch nationale Richter geleistet, weshalb sie auch als ,funktionale Gemeinschaftsrichter" bezeichnet werden können. ${ }^{46}$ Dabei dient das Vorabentscheidungsersuchen als Koordination zwischen den mitgliedstaatlichen Gerichten und dem EuGH. ${ }^{47}$ Deren Bedeutung ist für die Richtigkeitsgewähr bei der Anwendung des Unionsrechts nicht zu unterschätzen. ${ }^{48}$ Dieses Kooperationsverhältnis möchte der EuGH nicht gefährden. Die Staatshaftung bezweckt also vorrangig die Effektivität des Unionsrechts und den Rechtsschutz des Einzelnen und nicht die Sanktionierung einer Vorlagepflichtverletzung. ${ }^{49}$

Die soeben aufgezeigte Position des EuGH verdeutlicht auch die Rechtssache Kommission/Italien, ${ }^{50}$ wenngleich sie kein Fall der unionsrechtlichen Staatshaftung ist: In dem Vertragsverletzungsverfahren ging es um ein neu eingeführtes italienisches Gesetz zur Erstattung unionsrechtswidrig erhobener Abgaben, welches das für mit dem Unionsrecht unvereinbar erklärte Vorgängergesetz ablöste. Das neue Gesetz war für sich genommen mit dem Unionsrecht vereinbar. Allerdings wurde es von der italienischen Verwaltung und Rechtsprechung weiterhin in unionsrechtswidriger Weise ausgelegt und angewandt. Daher leitete die Kommission ein Vertragsverletzungsverfahren nach Art. 258 AEUV ein. Obgleich ein Unionsrechtsverstoß wegen fehlerhafter Auslegungspraxis der Verwaltung und der Gerichte festgestellt wurde, bildete das italienische Gesetz den Verfahrensgegenstand, wodurch der Unionsrechtsverstoß in der Legislative verortet wurde. Grundsätzlich hätten auch die Gerichtsentscheidungen zu einer Vertragsverletzung führen können, denn diese kann unabhängig davon festgestellt werden, welches Staatsorgan durch sein Handeln oder Unterlassen den Verstoß verursacht hat, selbst wenn es sich um ein verfassungsmäßig unabhängiges Organ handelt. ${ }^{51}$ Der bemerkenswerte Umweg sowohl der Kommission als auch des Gerichtshofs lässt sich damit erklären, dass diese Entscheidung nur drei Monate nach dem Urteil in der Rechtssache Köbler erging, welches bereits durch die Möglichkeit der Staatshaftung für unionsrechtswidrige Gerichtsentscheidungen den Druck auf die Höchstgerichte erhöhte. ${ }^{52}$ Zudem sieht die Kommission durch ein Vertragsverletzungsverfahren das Kooperationsverhältnis zwischen den mitgliedstaatlichen Gerichten und dem EuGH gefährdet und hält es zur effektiven Durchsetzung des Unionsrechts für ungeeignet. ${ }^{53}$ Indem die italienischen Gerichtsentscheidungen nur indirekt den Verfahrensgegen-

46 Schlussanträge GA Léger zu EuGH, Rs. C-224/01, Köbler, Slg. 2003, I-10239, Nr. 53 ff.; Radermacher, (Fn. 25), S. 1416.

47 Storr, (Fn. 43), S. 547; kritisch Radermacher, (Fn. 25), S. 1417.

48 Streinz, Europarecht-Staatshaftung für europarechtswidrige Gerichtsurteile - Staatshaftung, JuS 2004, S. 428; Schöndorf-Haubold, Die Haftung der Mitgliedstaaten für die Verletzung von EGRecht durch nationale Gerichte, JuS 2006, S. 114.

49 EuGH, Rs. C-224/01, Köbler, Slg. 2003, I-10239, Rdnr. 35; Schöndorf-Haubold, (Fn. 48), S. 114.

50 EuGH, Rs. C-129/00, Kommission/Italien, Slg. 2003, I-14637.

51 EuGH, Rs. 77/69, Kommission/Belgien, Slg. 1970, 237, Rdnr. 15; EuGH, Rs. C-129/00, Kommission/Italien, Slg. 2003, I-14637, Rdnr. 29.

52 Haltern, Verschiebungen im europäischen Rechtsschutzsystem, VerwArch 96 (2005), S. 327; ebenso Breuer, Urteile mitgliedstaatlicher Gerichte als möglicher Gegenstand eines Vertragsverletzungsverfahrens gem. Art. 226 EG?, EuZW 2004, S. 200, der aber auch den Grund in der schwierigen Umsetzung des Feststellungsurteils sieht.

53 Schlussanträge GA Tizzano zu EuGH, Rs. C-99/00, Lyckeskog, Slg. 2002, I-4839, Nr. 65; Breuer, (Fn. 52), S. 200. 
stand des Vertragsverletzungsverfahrens bildeten, wurde gleichzeitig die Rechtskraft der Gerichtsentscheidungen nicht in Frage gestellt und eine weitere Aushöhlung der mitgliedstaatlichen Rechtskraftprinzipien verhindert, wie sie in der Entscheidung Köbler praktiziert wurde. ${ }^{54}$

\section{Die Bestandskraftdurchbrechung gerichtlich bestätigter Verwaltungsentscheidungen}

Obgleich im Haftungsfall formal keine Änderung der mitgliedstaatlichen Gerichtsentscheidung vorliegt, wird die Wirkung der Rechtskraft nationaler Judikate und damit die Verfahrensautonomie begrenzt. Daran ändert auch die Tatsache nichts, dass der Gerichtshof die Haftungsvoraussetzungen restriktiv handhabt und sich des Kooperationsverhältnisses zu den nationalen Gerichten bewusst ist. Fraglich ist, ob sich der EuGH stets mit diesem Sekundärrechtsschutz zufrieden gibt und unter welchen Bedingungen das Unionsrecht die Korrektur der rechtskräftigen Entscheidung verlangt. In einer gefestigten Rechtsprechung hat der EuGH Kriterien entwickelt, anhand derer ein unionsrechtswidriger Verwaltungsakt aufgehoben werden muss oder das Vertrauen in die Verwaltungsentscheidung geschützt ist. ${ }^{55}$ Auch die Bestandskraft leistet einen Beitrag zur Rechtssicherheit. Können die Voraussetzungen zur Bestandskraftdurchbrechung also auf die Rechtskraft übertragen werden?

$\mathrm{Zu}$ dieser Frage äußerte sich der Gerichtshof in der Rechtssache Kühne \& Heitz, ${ }^{56}$ in der es um die Bestandskraft eines unionsrechtswidrigen, aber rechtskräftig gerichtlich bestätigten Verwaltungsaktes ging, nur am Rande. Der niederländische Geflügelexporteur Kühne \& Heitz hatte erfolglos gegen einen Rückforderungsbescheid geklagt, wobei das niederländische letztinstanzliche Gericht entgegen seiner Pflicht aus Art. 267 Abs. 3 AEUV von einer Vorlage an den EuGH abgesehen hatte. In einer anderen Rechtssache entschied der EuGH über die Auslegung der einschlägigen unionsrechtlichen Bestimmungen so, wie es dem Interesse von Kühne \& Heitz entsprach. Daraufhin verlangte Kühne \& Heitz die Rückzahlung der erstatteten Beträge von der Behörde. Das mit der Angelegenheit befasste Gericht wandte sich mit der Frage an den EuGH, ob die Behörde zur Rücknahme des bestandskräftigen Bescheids verpflichtet sei. Dieser ging im vorliegenden Fall zwar aufgrund bestimmter Kriterien von einer Überprüfungspflicht des Verwaltungsakts der Be-

54 Kremer, (Fn. 12), S. 479.

$55 \mathrm{EuGH}$, verb. Rs. 7/56 und 3-7/57, Algera, Slg. 1957, 87, 118; EuGH, verb. Rs. 42/59 und 49/59, SNUPAT, Slg. 1961, 111, 173; EuGH, Rs. 14/61, Hoogovens, Slg. 1962, 511, 548; EuGH, Rs. 111/63, Lemmerz-Werke, Slg. 1965, 93, 911.

56 EuGH, Rs. C-453/00, Kühne \& Heitz, Slg. 2004, I-837. 
hörde aus, diese Verpflichtung bestände aber gerade nicht generell. ${ }^{57}$ Damit beachtete er die grundsätzliche Verfahrensautonomie der Mitgliedstaaten. ${ }^{58}$ Das unanfechtbare Urteil erwähnte er lediglich bei der Sachverhaltsschilderung, weshalb seine Entscheidung vielmehr die Bestandskraft zum Gegenstand hatte als die Aufhebung oder Korrektur des nationalen Urteils. ${ }^{59}$ Generalanwalt Léger ging hingegen ausdrücklich auf die Rechtskraftproblematik ein: Es sei nicht maßgeblich, ob ein rechtskräftiges Urteil vorliegt, denn wie schon in der Rechtssache Köbler könne im Hinblick auf den Vorrang des Unionsrechts eine nationale Verfahrensvorschrift keine unionsrechtliche Schadensersatzklage wegen einer unionsrechtswidrigen Gerichtsentscheidung verhindern. ${ }^{60}$ Auch in Teilen der Literatur wird die Entscheidung Kühne \& Heitz mit der Staatshaftungsrechtsprechung des EuGH in Verbindung gebracht, da auch durch diese Entscheidung die nationalen Gerichte zur Vorlage an den EuGH veranlasst werden sollten. ${ }^{61}$ Für den Gerichtshof war in der Rechtssache Kühne \& Heitz tatsächlich ausschlaggebend, dass das letztinstanzliche Gericht seine Pflicht aus Art. 267 AEUV verletzt hatte. Als Voraussetzung für die Überprüfungs- und Rücknahmepflicht der Verwaltungsbehörde kam es ihm allerdings auch entscheidend darauf an, dass eine Rücknahme nach nationalem Recht möglich war und er folgte damit nicht der Auffassung des Generalanwalts. Der EuGH lässt hier bewusst offen, ob die Voraussetzungen zur Bestandskraftdurchbrechung auf eine mögliche Rechtskraftdurchbrechung zu übertragen sind.

Dies musste er dann in der Rechtssache Kapferer $^{62}$ entscheiden, obwohl es hier nicht um einen gerichtlich bestätigten Verwaltungsakt ging. In dem Rechtsstreit hatte ein österreichisches erstinstanzliches Gericht eine EU-Verordnung falsch ausgelegt und sich für international zuständig erklärt. Das nationale Verfahrensrecht bot dem zweitinstanzlichen

57 Eine Verwaltungsbehörde ist zur Überprüfung einer bestandskräftigen Verwaltungsentscheidung verpflichtet, wenn (1.) die Behörde nach nationalem Recht zur Rücknahme befugt ist, (2.) die Entscheidung infolge eines letztinstanzlichen nationalen Gerichtsurteils bestandskräftig geworden ist, (3.) das Urteil, wie eine nach seinem Erlass ergangene EuGH-Entscheidung zeigt, auf einer unrichtigen Auslegung des Unionsrechts beruht und entgegen Art. 267 Abs. 3 AEUV ohne Vorabentscheidung des Gerichtshofs erfolgt ist und (4.) der Betroffene sich unmittelbar nachdem er Kenntnis von der besagten EuGH-Entscheidung erlangt hat, an die Verwaltungsbehörde gewandt hat, vgl. EuGH, Rs. C-453/00, Kühne \& Heitz, Slg. 2004, I-837, Rdnr. 28. Inwieweit diese Voraussetzungen abstrakt-genereller Natur sind, ist jedoch fraglich, da der Gerichtshof in dem ähnlich gelagerten Fall EuGH, Rs. C-2/06, Kempter, Slg. 2008, I-411, die zur Aufhebung der Verwaltungsentscheidung führenden Umstände anders gewichtete und fortentwickelte, siehe Kanitz/Wendel, Gemeinschaftsrechtlich gebotene Grenzen der Bestandskraftdurchbrechung im europäischen Verwaltungsverfahren?, Zur Frage prozessualer Vorbedingungen und zeitlicher Schranken der Überprüfungspflicht bestandskräftiger Verwaltungsakte, EuZW 2008, S. 234 f.

58 Vgl. Kremer, (Fn. 12), S. 482 f.

59 Dazu Schmidt-Westphal/Sander, Anmerkung, EuZW 2006, S. 243. Gleichwohl kann die Bestandskraftdurchbrechung im nationalen Recht unter Umständen nur durch eine Rechtskraftdurchbrechung bewirkt werden, so etwa im englischen Recht nach Part 52.17 Civil Procedure Rules 1998, siehe Feakins and another v. Intevention Board for Agricultural Produce [2006] EWAC Civ 699. Im deutschen Recht ist die Rücknahme des Verwaltungsakts ohne Rechtskraftdurchbrechung nach $\S \S 48,51$ Abs. 5 VwVfG möglich.

60 Schlussanträge GA Léger zu EuGH, Rs. C-453/00, Kühne \& Heitz, Slg. 2004, I-837, Nr. 66, Fn. 56.

61 Kenntner, (Fn. 43), S. 237; Kremer, (Fn. 12), S. 485.

62 EuGH, Rs. C-234/04, Kapferer, Slg. 2006, I-2485. 
Berufungsgericht keine Möglichkeit, die rechtskräftige Zuständigkeitsentscheidung zu korrigieren, weshalb sich die Frage nach der Rechtskraftdurchbrechung stellte. Der EuGH verlangte dies unter Bezug auf die Bedeutung des Rechtskraftprinzips nicht. Zur Übertragbarkeit der Kühne \& Heitz-Kriterien zur Bestandskraftdurchbrechung auf die Rechtskraft führte er aus:
„Selbst wenn [...] die in diesem Urteil aufgestellten Grundsätze auf einen Sachverhalt [wie diesen] übertragbar sein sollten, [...] ist doch zu beachten, dass dieses Urteil die Verpflichtung der betreffenden Behörde aus Art. 10 EG [Art. 4 Abs. 3 EUV], eine unter Verstoß gegen Ge- meinschaftsrecht erlassene bestandskräftige Entscheidung zu überprüfen, u.a. von der Voraus- setzung abhängig macht, dass diese Behörde nach nationalem Recht zur Rücknahme dieser Entscheidung befugt ist. Im vorliegenden Fall ist aber diese Voraussetzung [...] nicht er- füllt". 63

Diese Aussage wird in der Literatur so interpretiert, dass der EuGH der grundsätzlichen Frage nach der Übertragbarkeit ausgewichen und die Rechtskraft hier mangels Korrekturmöglichkeiten des nationalen Verfahrensrechts anzuerkennen sei. ${ }^{64} \mathrm{Im}$ Ergebnis werden also die Voraussetzungen der Rechtssache Kühne \& Heitz angewendet. Deutlicher wird die Position des EuGH in den Schlussanträgen von Generalanwalt Tizzano:
„Eine Frage anderer Natur und Tragweite [als die Rücknahme von Verwaltungsentscheidun- gen] ist jedoch die Frage nach dem Grundsatz der Rechtskraft, also einem Grundprinzip, das allein gerichtliche Entscheidungen kennzeichnet. Ich bin daher nicht der Ansicht, dass sich die Lösungen, zu denen der Gerichthof in diesem Urteil gelangt ist, ohne weiteres einfach [...] übertragen lassen“" ${ }^{65}$

Obgleich die Bestandskraft und Rechtskraft beide einen Beitrag zur Rechtssicherheit leisten und zum Beispiel im deutschen und österreichischen Recht gemeinsame Wurzeln haben, ${ }^{66}$ kommt der Rechtskraft die besondere Funktion zu, die endgültige Befriedigung allein nach Maßgabe des Rechts zu schaffen. Im Gegensatz zu von einem neutralen Richter gefällten rechtskräftigen Urteilen können die Entscheidungen der am Rechtsverhältnis beteiligten Verwaltung ohne gerichtliche Prüfung dauerhaft werden. ${ }^{67}$ Deshalb sind Verwaltungsakte mit geringerer Beständigkeit ausgestattet als Urteile. Die Bestandskraft legitimiert sich allein aus der administrativen Funktionalität. ${ }^{68}$ Hinzu kommt, dass die materielle Rechtskraft neben die Bindungswirkung einer Verwaltungsentscheidung tritt, dieser aber keine andere Qualität verleiht. ${ }^{69}$ Die beiden Subprinzipien der Rechtssicherheit

63 Ibid., Rdnr. 23.

64 Schmahl/Köber, Durchbrechung der Rechtskraft nationaler Gerichtsentscheidungen zu Gunsten der Effektivität des Unionsrechts?, EuZW 2010, S. 929; Ludwigs, Der Schutz der Rechtskraft im Gemeinschaftsrecht, ZfRV 2006, S. 193; König, (Fn. 15), S. 181 f.

65 Schlussanträge GA Tizzano zu EuGH, Rs. C-234/04, Kapferer, Slg. 2006, I-2485, Nr. 25.

66 Zum deutschen Recht: Ruffert, Anmerkung, JZ 2006, S. 905; Maurer, Allgemeines Verwaltungsrecht, 18. Aufl. 2011, § 11, Rdnr. 3; zum österreichischen Recht: von Arnauld, (Fn. 8), S. 279; Hatje, in: Holoubek/Lang, Das EuGH-Verfahren in Steuersachen, 2000, S. 135.

67 Vgl. von Arnauld, (Fn. 8), S. 279, 508.

68 Ludwigs, (Fn. 64), S. $193 \mathrm{f}$.

69 Ibid., S. 193. 
sind daher nicht wertungsgleich und lassen sich nicht stets in gleicher Weise lösen. ${ }^{70}$ Die „Kühne \& Heitz“-Kriterien müssen daher nicht ohne Weiteres auf Fälle der Rechtskraftdurchbrechung übertragen werden. Zudem hätte es dann letztlich das nationale Recht in der Hand, seinen Rechtsinstituten der Rechts- und Bestandskraft gegenüber dem Unionsrecht absolute Geltung zu verschaffen. ${ }^{71}$ Dies zeigt, dass eine Übertragung auf gerichtliche Verfahren nicht in allen Fällen möglich ist.

Generalanwalt Tizzano prüfte nur vorsorglich die Voraussetzungen zur Bestandskraftdurchbrechung, von denen aber keine erfüllt waren. ${ }^{72}$ Daher bleibt die Rechtskraft der österreichischen erstinstanzlichen Zuständigkeitsentscheidung im vorliegenden Fall auch bestehen, wenn die Kriterien aus Kühne \& Heitz angewendet werden würden. So ist auch der Hinweis des Gerichtshofs zu verstehen, diesem Ergebnis stünde auch das Urteil Kühne \& Heitz nicht entgegen. ${ }^{73}$ Der EuGH nimmt in der Rechtssache Kapferer eine Abwägung zwischen der Verfahrensautonomie und dem Äquivalenz- und Effektivitätsgebot vor. Diese erfolgte hier zutreffend zugunsten der Rechtskraft, da der EuGH die nationalen Rechtskraftprinzipien als Ausdruck der Rechtssicherheit anerkennt und dementsprechend einen großzügigen Maßstab anlegt. Die effektive Durchsetzung des Unionsrechts kann nicht stets die Opferung der Rechtskraft rechtfertigen. ${ }^{74}$ Die Anwendung der Vorrangregel wie es Generalanwalt Léger in der Rechtssache Kühne \& Heitz forderte, ist für Fälle gerichtlich bestätigter Verwaltungsakte in der EuGH-Rechtsprechung nicht erkennbar.

\section{Kollisionen im Beihilfen- und Steuerrecht}

Von diesem großzügigen Maßstab weicht der EuGH im Bereich des Beihilfenrechts ab und verdeutlicht, dass die nationalen Rechtskraftprinzipien nicht absolut geschützt sind. So konnte sich in der Rechtssache Lucchini ${ }^{75}$ ein rechtskräftiges italienisches Gerichtsurteil, welches die Rechtmäßigkeit einer Beihilfe feststellte, gegen die zuvor ergangene Verbotsentscheidung der Kommission nicht durchsetzen. Italienische Zivilgerichte hatten die zuständigen Behörden rechtskräftig zur Auszahlung von Beihilfen an das Unternehmen Lucchini verpflichtet, woraufhin die entsprechenden Beträge ausgezahlt wurden. Zuvor waren die Beihilfen von der Kommission für unvereinbar mit dem gemeinsamen Markt erklärt worden, was die zivilgerichtlichen Urteile jedoch nicht berücksichtigten. Die Kommission wies die Behörden an, die Beihilfen zurückzufordern. Dem kamen die Behörden nach. Das mit der Anfechtung des Widerrufs befasste Verwaltungsgericht sah sich aufgrund einer italienischen Verfahrensvorschrift zur Rechtskraft an die Feststellung der Zivilgerichte über die Rechtmäßigkeit der Beihilfen gebunden. Folglich hätte die Kommissionsentscheidung in dem Verwaltungsgerichtsverfahren nicht berücksichtigt werden können. Das Zivilgericht hatte aber durch seine Feststellung in die primärrechtliche Kompetenzverteilung eingegriffen, da allein die Kommission nach Art. 108 AEUV über die Verein-

70 Germelmann, (Fn. 2), S. 264; Ruffert, (Fn. 66), S. 905.

71 Germelmann, (Fn. 2), S. 271.

72 Schlussanträge GA Tizzano zu EuGH, Rs. C-234/04, Kapferer, Slg. 2006, I-2485, Nr. 26 ff.

73 EuGH, Rs. C-234/04, Kapferer, Slg. 2006, I-2485, Rdnr. 23.

74 Schmidt-Westphal/Sander, (Fn. 59), S. 243; Ludwigs, (Fn. 64), S. 194.

75 EuGH, Rs. C-119/05, Lucchini, Slg. 2007, I-6199. 
barkeit einer staatlichen Beihilfe mit dem gemeinsamen Markt entscheidet. Angesichts dieses Konflikts zwischen der Verfahrensautonomie und dem unmittelbar anwendbaren Unionsrecht, wandte sich das Verwaltungsgericht mit einem Vorabentscheidungsersuchen an den Gerichtshof. Dieser entschied, dass die italienische Rechtskraftvorschrift der Wirksamkeit des Unionsrechts entgegenstünde und daher unionsrechtskonform auszulegen sei oder erforderlichenfalls unangewendet bleiben müsse.

Die Rechtssache Lucchini ist der erste Fall, in dem der Gerichtshof die Rechtskraft einer nationalen Gerichtsentscheidung direkt in Frage stellt. Auffallend ist, dass der EuGH im Gegensatz zu seiner vorhergehenden Rechtsprechung und den Ausführungen des Generalanwalts auf die Bedeutung der Rechtskraft und das Verhältnis der nationalen Rechtskraftprinzipien zum Unionsrecht nicht eingeht. Zudem nimmt er auf seine bisherige Rechtskraftrechtsprechung keinen Bezug, sondern weist auf seine Entscheidungen Simmenthal und Factortame I hin. ${ }^{76}$ In diesen Entscheidungen ging er von einer direkten Kollision der unionsrechtlichen Vorschriften mit den nationalen Regelungen aus, weshalb dem Unionsrecht unstreitig der Vorrang zukam. In der Rechtssache Lucchini nahm der Gerichtshof eine direkte Kollision der Rechtskraftvorschrift mit der primärrechtlichen Regelung des Art. 108 AEUV zur Beihilfenaufsicht an und wandte die Vorrangregel an. Da das rechtskräftige Zivilurteil der Wirksamkeit des Unionsrechts entgegenstand, musste konsequenter Weise die Rechtskraftwirkung korrigiert werden. ${ }^{77}$ Diese Vorgehensweise wirft die Frage auf, ob die Rechtskraft bei Zuständigkeitsmängeln generell durchbrochen werden muss oder lediglich ein außergewöhnlicher Fall des Beihilfenrechts vorliegt. ${ }^{78}$ Nach dem englischen „res judicata“-Prinzip werden Entscheidungen von unzuständigen Gerichten generell die Rechtskraftwirkung abgesprochen. ${ }^{79}$ Bei der Übertragung dieses Prinzips auf das Unionsrecht müsste berücksichtigt werden, dass sich die sachliche Zuständigkeit der nationalen und unionsrechtlichen Gerichte mangels hinreichender Klarheit des EU-Vertrages nicht immer trennscharf bestimmen lässt und daher vom EuGH vorgenommen werden muss. ${ }^{80}$ Das italienische Zivilgericht in der Rechtssache Lucchini war nicht absolut unzuständig, sondern hatte nur keine Bewertungszuständigkeit hinsichtlich der Vereinbarkeit der Beihilfe mit dem Gemeinsamen Markt. ${ }^{81}$ Daher kann das Prinzip res judicata nicht auf das Unionsrecht übertragen werden. Die Rechtskraftdurchbrechung ist zwar grundsätzlich nicht ausgeschlossen, die Entscheidung Lucchini stellt jedoch eine Ausnahme dar, die

76 Ibid., Rdnr. 61; EuGH, Rs. 106/77, Simmenthal, Slg. 1978, 629; EuGH, Rs. C-213/89, Factortame I, Slg. 1990, I-2433.

77 Kremer, (Fn. 12), S. 488.

78 Germelmann, Neue Wege in der Rechtsprechung des EuGH zu nationalen Rechtskraftregeln?, EWS 2007, S. 395 f.

79 R v. Hutchings (1881) 6 QBD 300; Wakefield Cpn. v. Cooke [1994] AC 31; Spencer Bower/ Turner, The doctrine of res judicata, 2. Aufl. 1969, S. 92.

80 Germelmann, (Fn. 78), S. 396.

81 Ibid.; Haratsch/Hensel, Anmerkung, JZ 2008, S. 144. 
„nur in ganz besonderen Fällen zulässig [ist], in denen der für die betroffenen Parteien geltende Spruch,res iudicatas pro veritate habetur" einem schwerer wiegenden Rechtsinteresse weichen muss". 82

Den Ausnahmecharakter dieses Falls bestätigt das Vertragsverletzungsverfahren in der Rechtssache Kommission/Slowakische Republik. ${ }^{83}$ Ein überschuldeter slowakischer Alkoholhersteller schloss mit seinen Gläubigern einen gerichtlichen Vergleich ab. Dadurch sollte ähnlich wie in einem Insolvenzverfahren die finanzielle Situation des Unternehmens geregelt, aber gleichzeitig die Fortsetzung seiner Tätigkeit gewährleistet werden. In dem rechtskräftigen Vergleich erließ das Finanzamt dem Unternehmen Alkoholverbrauchssteuern. Die Kommission stellte fest, dass der Steuererlass eine unionsrechtswidrige Beihilfe darstelle und vom Finanzamt zurückgefordert werden müsse. Da ihre Entscheidung nicht sofort vollstreckt wurde, leitete sie ein Vertragsverletzungsverfahren ein. Den Verfahrensgegenstand bildete das Unterlassen der slowakischen Behörde. Der EuGH hatte nun den umgekehrten Fall zu entscheiden: Das rechtskräftige Urteil des slowakischen Gerichts, welches die Behörden zur Gewährung einer Beihilfe verpflichtete, erging vor der Verbotsentscheidung der Kommission. Im Gegensatz zur Kommission hielt der Gerichtshof das Urteil in der Rechtssache Lucchini nicht für einschlägig und stellte eine Verbindung zu seinen Entscheidungen Köbler, Kapferer und Olimpiclub her. Aufgrund der Bedeutung der Rechtskraft sei eine Rechtskraftdurchbrechung nicht zwingend erforderlich, auch wenn dann ein unionsrechtswidriger Zustand bestehen bleibt. Die Rückforderungsentscheidung der Kommission genießt somit keinen Absolutheitsanspruch. ${ }^{84}$ Sie kann sich bei einer indirekten Kollision mit den nationalen Rechtskraftregelungen nur als Ergebnis der Abwägung zwischen dem Effektivitätsprinzip und der Verfahrensautonomie durchsetzen.

Nach slowakischem Recht hätte die Rückforderung in zwei Schritten erfolgen müssen: zunächst die Aufhebung der beihilfengenehmigenden Gerichtsentscheidung und erst danach die Rückforderung der Beihilfe selbst. ${ }^{85}$ Nach nationalem Recht standen entsprechende Möglichkeiten zur Verfügung, blieben aber ungenutzt. Das Unterlassen genügte für eine Vertragsverletzung. Ob das nationale Recht einen rechtskraftdurchbrechenden Rechtsbehelf bereithält, spielt bei der Frage nach der Durchsetzung der rechtskräftigen nationalen Gerichtsentscheidung gegenüber der Rückforderungsentscheidung aber keine Rolle. ${ }^{86} \mathrm{Wie}$ bereits die Entscheidungen Kühne \& Heitz und Kapferer gezeigt haben, sind die Erfordernisse zur Bestandskraftdurchbrechung nicht auf Fälle der Rechtskraftdurchbrechung zu übertragen. Generalanwalt Cruz Villalón machte deshalb in seinen Schlussanträgen auf die Bedeutung der Ausweitung des Staatshaftung auf Gerichtsentscheidungen im Gesamtgefüge des europäischen Rechtsschutzsystems aufmerksam: Für den Fall, dass eine Korrektur

82 Schlussanträge GA Geelhoed zu EuGH, Rs. C-119/05, Lucchini, Slg. 2007, I-6199, Nrn. 16, 46; auch in der Literatur wird Lucchini als Ausnahmefall betrachtet, siehe Kremer, (Fn. 12), S. 488; ders., Effektuierung des europäischen Beihilfenrechts durch die Begrenzung der Rechtskraft, EuZW 2007, S. 729; Germelmann, (Fn. 78), S. 397 f.

83 EuGH, Rs. 507/08, Kommission/Slowakische Republik, Slg. 2010, I-13489.

84 Bartosch, Die EU-Beihilfenkontrolle vor der nationalen Gerichtsbarkeit, RIW 2011, S. 585.

85 Koenig, Der EU-beihilfenrechtliche Effektivitätsgrundsatz im nationalen Prozessrecht, EuZW 2011, S. 703.

86 So allerdings Becker, Die parallele Prüfung beihilfenrechtlicher Sachverhalte durch Kommission und nationale Gerichte - Entscheidungskompetenzen und -konflikte, EuZW 2012, S. 729. 
des rechtskräftigen Urteils nicht möglich sei, könne die Entschädigungshaftung greifen. ${ }^{87}$ Dazu muss es jedoch nicht unbedingt kommen, da sich bei indirekten Kollisionen eine Lösung zugunsten der unionsrechtlichen Effektivität höchstens als Ergebnis der Abwägung ergibt. Aus beihilfenrechtlicher Sicht wird dies teilweise kritisiert, da die Besonderheit bestehe, dass der Beihilfenempfänger nur dann auf den Bestand der Beihilfe vertrauen darf, wenn das Verfahren nach Art. 108 AEUV eingehalten wurde. ${ }^{88}$ Hiergegen ist anzuführen, dass die Parteien eines Rechtsstreits im (auch öffentlichen) Interesse der Rechtssicherheit und des Rechtsfriedens auf die Rechtskraft vertrauen können müssen. Zudem sind die Kriterien zur Gewährung von Vertrauensschutz auf den Bestand einer Verwaltungsentscheidung gerade nicht auf die Rechtskraft zu übertragen.

Im Ergebnis konnte sich die Rechtskraft in der Entscheidung Kommission/Slowakische Republik nicht durchsetzen. Auch in der Rechtssache Olimpiclub im Bereich des nur teilharmonisierten Mehrwertsteuersystems, konnte der Eingriff in die italienische Rechtskraftvorschrift durch das Effektivitätsprinzip gerechtfertigt werden. ${ }^{89}$ Ein italienisches Gericht hatte darüber zu entscheiden, ob eine missbräuchliche Praxis einer Kapitalgesellschaft auf dem Gebiet der Mehrwertsteuer vorlag und Steuererklärungen korrigiert werden mussten. Eine in diesem Rechtsstreit relevante Vorfrage war bereits rechtskräftig entschieden worden. Das Gericht fühlte sich einerseits durch das italienische Verfahrensrecht an diese Feststellung gebunden, dies hätte aber andererseits dazu geführt, dass sein Urteil gegen Unionsrecht verstößt. Daher wird in Olimpiclub kein rechtskräftiges Urteil unmittelbar in Frage gestellt, vielmehr geht es um die Reichweite der Rechtskraft. Das Gericht legte dem EuGH die Frage zur Vorabentscheidung vor, ob das Unionsrecht auch in anderen Bereichen als dem der staatlichen Beihilfen der Anwendung einer Rechtskraftvorschrift entgegenstehe. Der Gerichtshof bejahte dies, nachdem er die Verfahrensautonomie mit dem unionsrechtlichen Effektivitätsgebot abgewogen hatte. Ausdrücklich grenzte er die Rechtssache Olimpiclub von seiner Entscheidung Lucchini ab, die einen Bereich der ausschließlichen Unionskompetenzen betroffen hatte und so ein besonderer Sachverhalt zugrunde lag. ${ }^{90}$

Die EuGH-Rechtsprechung zum Beihilfenrecht und Mehrwertsteuersystem zeigt, dass die mitgliedstaatlichen Rechtskraftprinzipien nicht so unantastbar sind, wie es die Rechtssache Kapferer vermuten lässt. ${ }^{91}$ Der EuGH weicht hier von seinem großzügigen Maßstab ab. Das Effektivitätsgebot kann tatsächlich die Durchbrechung der Rechtskraft rechtfertigen. Auch die Bindungswirkung nationaler Rechtskraftregelungen ist im Verhältnis zum

87 Schlussanträge GA Cruz Villalón zu EuGH, Rs. C-507/08, Kommission/Slowakische Republik, Slg. 2010, I-13489, Nr. 47.

88 Becker, (Fn. 86), S. 729.

89 EuGH, Rs. C-2/08, Olimpiclub, Slg. 2009, I-7501, Rdnrn. 28, 32.

90 Ibid., Rdnr. 25.

91 Gewisse Parallelen lassen sich zu den Entscheidungen zur Rechtskraft unionsrechtswidriger Schiedssprüche in EuGH, Rs. C-40/08, Asturcom, Slg. 2009, I-9579 und EuGH, Rs. C-126/97, Eco Swiss, Slg. 1999, I-3055 ziehen. Auf letztere nimmt der EuGH zur Begründung seiner Position in Kapferer Bezug. Die Aspekte der Rechtskraft und Effizienz des Schiedsverfahrens werden in die Abwägung gegenüber der Effektivität des Unionsrechts einbezogen, die Rechtskraftdurchbrechung ist jedoch nicht ausgeschlossen. Die Ausweitung der Rechtskraftrechtsprechung auf die Schiedsgerichtsbarkeit ist aber wegen der fehlenden Vorlagemöglichkeit gem. Art. 267 AEUV problematisch, vgl. Schmahl/Köber, (Fn. 64), S. 932. 
unionsrechtlichen Effektivitätsgebot abwägungserforderlich. ${ }^{92}$ Andererseits verdeutlichen die vorliegenden Entscheidungen auch, dass für Bereiche außerhalb der normativen Kompetenz der Union, die Wirksamkeit des EU-Rechts nicht absolut gewährleistet werden muss und zeichnen damit ein zwiespältiges Bild. Je stärker ein Zuständigkeitsbereich der Union tangiert wird, desto wahrscheinlicher ist es, dass die Rechtskraft einer nationalen Gerichtsentscheidung bzw. deren Bindungswirkung durchbrochen werden muss.

\section{E. Die Auswirkungen auf das Rechtskraftprinzip der Mitgliedstaaten}

Obgleich der EuGH die Verfahrensautonomie der Mitgliedstaaten anerkennt, können Eingriffe durch das Effektivitätsgebot in Ausnahmefällen gerechtfertigt sein. In diesem Fall folgt aus der Verpflichtung der Mitgliedstaaten zur Durchführung des Unionsrechts aus Art. 4 Abs. 3 EUV, dass sie das nationale Verfahrensrecht unionsrechtskonform auslegen oder unangewendet lassen. Dies kann in vielen Konstellationen relevant werden: Zum Beispiel könnte die Bindungswirkung einer rechtskräftigen Gerichtsentscheidung ein anderes Gericht dazu veranlassen, eine unionsrechtswidrige Entscheidung zu treffen. ${ }^{93}$ Von den nationalen Richtern wird dann eine dem Unionsrecht entsprechende Auslegung der Rechtskraftvorschrift gefordert, soweit die äußerste Grenze des Verbots einer contra legem Auslegung eingehalten wird. ${ }^{94}$ Ist eine unionsrechtswidrige Gerichtsentscheidung ergangen, bietet möglicherweise das nationale Recht entsprechende Rechtsbehelfe, um das rechtskräftige Urteil zu korrigieren und einen unionsrechtskonformen Zustand herzustellen. Das Unionsrecht verlangt dies von den Mitgliedstaaten jedoch nicht. Ausreichend ist ein Staatshaftungsverfahren, durch das die Schäden der Fehlentscheidung beseitigt werden können. Daher soll im Folgenden am Beispiel des deutschen und englischen Rechts untersucht werden, ob das mitgliedstaatliche Verfahrensrecht einen ausreichenden Primär- oder Sekundärrechtsweg zu Verfügung stellt. ${ }^{95}$

Primärrechtsbehelfe zur Korrektur einer rechtskräftigen Gerichtsentscheidung sind in den Mitgliedstaaten auf verschiedenste Weise geregelt, was auf ihr unterschiedliches Verständnis der Funktionen der Rechtskraft zurückzuführen ist.

So soll im deutschen Recht einerseits verhindert werden, dass gerichtliche Erkenntnisse zeitlich unbegrenzt aufgehoben oder abgeändert werden können (formelle Rechtskraft), andererseits wird für die Parteien der Inhalt der Entscheidung maßgeblich (materielle Rechtskraft). ${ }^{96}$ Die formelle Rechtskraft tritt ein, wenn gegen die Entscheidung keine ordentlichen Rechtsmittel mehr gegeben sind und ist Voraussetzung für den Eintritt der materiellen Rechtskraft. Danach kann die Entscheidung nur mithilfe gesetzlich normierter, eng begrenzter außerordentlicher Rechtsbehelfe korrigiert werden.

92 Ibid., S. 931.

93 Diese Situation ähnelt Kapferer, Lucchini und Olimpiclub.

94 Vgl. Galetta, (Fn. 14), S. 43.

95 Fraglich ist, ob daneben ein Vertragsverletzungsverfahren nach Art. 258 AEUV wegen einer einzelnen Fehlentscheidung möglich ist. Dies grundsätzlich bejahend Breuer, (Fn. 52), S. 201, der aber davon ausgeht, dass von einer im Ermessen der Kommission stehenden Klageerhebung gegen einen Mitgliedstaat in der Praxis abgesehen wird.

96 Rosenberg/Schwab/Gottwald, Zivilprozessrecht, 17. Aufl. 2010, § 150, Rdnr. 1 f. 
Dem englischen Recht ist dagegen eine solche Unterscheidung der Rechtsbehelfe fremd. Eine Gerichtsentscheidung kann nur „,res judicata“-Wirkung entfalten, wenn folgende Voraussetzungen vorliegen:

„It must be pronounced by a judicial tribunal, of competent jurisdiction, and the decision itself must be a judicial decision that is final and conclusive and on the merits" ${ }^{97}$

Andernfalls ist die Entscheidung void und entfaltet keine Bindungswirkung. Die Gültigkeit der Entscheidung kann mittels collateral attacks überprüft werden. ${ }^{98}$ Die Voraussetzung einer final and conclusive decision ist erfüllt, wenn die Gerichtsentscheidung die Rechtsfrage im Verfahren erörtert hat und abschließend entscheiden wollte. ${ }^{99}$ Unanfechtbarkeit ist keine Tatbestandsvoraussetzung. Daher meint finality nicht non-reviewability. ${ }^{100}$ Das „res judicata“-Prinzip entfaltet dadurch eine sehr weitgehende Wirkung, ist aber auch mit kollidierenden Prinzipien und Interessen wie der Verfahrensökonomie, dem Individualrechtsschutz, dem Rechtsfrieden und der materiellen Gerechtigkeit abzuwägen und so für Durchbrechungen offen. Dies verleiht dem Prinzip res judicata Flexibilität um seine Zielsetzungen berücksichtigen zu können. ${ }^{101}$ Die Durchbrechungsmöglichkeiten wurden in ständiger Rechtsprechung entwickelt und lassen sich grob zwei Situationen zuordnen: Die Nichtanwendung des „res judicata“-Prinzips kann durch die Beachtung der statutory duties oder aufgrund special circumstances and special cases geboten sein.

Dass von der Judikative und Exekutive die statutory duties beachtet werden müssen, folgt aus dem Prinzip der parliamentary sovereignty. Indem allein das Parlament die Kompetenz zur Ausübung staatlicher Gewalt festlegen kann, soll verhindert werden, dass die Judikative ihre Kompetenzen selbständig ausweitet. Die aus der Parlamentssouveränität abgeleiteten statutory duties enthalten das Verbot, eine Situation durch Gerichtsentscheidungen zu verfestigen, die einer gesetzlichen Vorschrift entgegensteht. ${ }^{102}$ Aufgrund des scheinbaren Widerspruchs zu dem Grundsatz, dass auch inhaltlich falsche Entscheidungen „res judicata“"-Wirkung entfalten, muss weiter differenziert werden: Die Gerichtsentscheidung kann den statutory duties tatsächlich widersprechen oder die gesetzlichen Vorgaben

97 Barnett, Res judicata, estoppel, and foreign judgements, 2001, S. 11.

98 Herzog/Karlen, Attacks on Judicial Decisions, in: Capelletti (Hrsg.), Civil Procedure, International Encyclopedia of Comparative Law, Bd. 16, Chapter 8, 1982, Rdnr. 2.

99 Barnett, (Fn. 97), S. 16.

100 Zeuner/Koch, Effects of Judgment (Res Judicata), in: Capelletti, (Fn. 98), Chapter 9, 2012, Rdnrn. 13, 19; Colt Industries Inc v. Sarlie (No 2) [1966] 1 WLR 1287 (CA); Railways Comr (NSW) v. Cavanough (1935) 53 CLR 220 (HCA).

101 Vgl. Carl Zeiss Stiftung v. Rayner \& Keeler (No 2) [1967] 1 A.C. 853, 947: „It is open to the courts to recognise that in special circumstances inflexible application of it [the docrtine of res judicata] may have the opposite result".

102 In re Warting Westminster Bank v. Burton-Butler [1948] 1 Ch. 221; Germelmann, (Fn. 2), S. 250. Dies ist besonders für öffentlich-rechtliche Fälle relevant, da Gerichtsentscheidungen den betroffenen Hoheitsträger an der Erfüllung seiner gesetzlichen Verpflichtungen hindern können. Daher wird das ,res judicata“-Prinzip durch die beiden Prinzipien begrenzt that jurisdiction cannot be exceeded and that statutory duties cannot be fettered und besitzt im öffentlichrechtlichen Prozessrecht somit eine geringe Bedeutung, Wade/Forsyth, Administrative Law, 7. Aufl. 1994, S. 277. 
etwa durch unzutreffende Auslegung lediglich falsch anwenden. ${ }^{103}$ Die Durchbrechungsmöglichkeit der statutory duties wird insgesamt restriktiv gehandhabt. ${ }^{104}$

Die Möglichkeit der special circumstances and special cases sah bereits die Entscheidung Henderson v. Henderson von 1843 vor, welche die Voraussetzungen beschreibt, unter denen eine gerichtliche Entscheidung als endgültig anzusehen ist. ${ }^{105}$ In welchen Situationen die besonderen Umstände oder speziell gelagerten Fälle die Ausnahme vom ,res judicata“Prinzip rechtfertigen, wird in dieser Entscheidung und in der weiteren Rechtsprechung nicht eindeutig herauskristallisiert. Ein durch fraud (Arglist) erlangtes Urteil kann einen solchen Fall begründen. ${ }^{106}$ Auch default judgments (Versäumnisurteile) können dazu führen. ${ }^{107}$ Tatsachen, die zum Zeitpunkt der Entscheidung bereits vorlagen, jedoch - aus welchen Gründen auch immer - nicht in das Verfahren eingebracht wurden, stellen keine Ausnahmesituation dar. Dies würde gegen das Prinzip der Streitkonzentration verstoßen. ${ }^{108}$ Über die special circumstances and special cases könnte auch einer unionsrechtlich gebotenen „res judicata“-Durchbrechung nachgekommen werden. In der Rechtssache Barber ${ }^{109}$ hat sich der Court of Appeal jedenfalls nicht grundsätzlich gegen eine solche Lösung ausgesprochen. Das House of Lords hatte in einem Parallelverfahren die Unvereinbarkeit einer nationalen arbeitsrechtlichen Gesetzesbestimmung mit dem Unionsrecht festgestellt. Auf diese Vorschrift hatte sich das rechtskräftige klageabweisende Urteil gegen die Klägerin gestützt. Diese erhob nun erneut Klage. Fraglich war, ob in diesem Fall das ,res judicata“Prinzip, insbesondere die Präklusion einer erneuten Klageerhebung, der Urteilskorrektur entgegenstand. In diesem Zusammenhang wurde die Möglichkeit angesprochen, im Falle der Unionsrechtswidrigkeit die Bindungswirkung des ,res judicata“-Prinzips zu durchbrechen. ${ }^{110}$ Der Fall konnte jedoch durch das nationale Recht gelöst werden, welches bereits der Anwendung der Präklusionsregel entgegenstand. Ob die special circumstances and special cases im englischen Recht tatsächlich zur unionsrechtlichen Rechtskraftdurchbrechung genutzt werden, kann erst die künftige Rechtsprechung zeigen.

Indem die deutsche Rechtskraftdogmatik die Grenzen für eine Abänderung des rechtskräftigen Urteils recht eng zieht, kann einer Durchbrechung im Falle der Unionsrechtswidrigkeit nur schwer nachgekommen werden. Eine rechtskräftige unionsrechtswidrige

103 Germelmann, (Fn. 2), S. $251 \mathrm{f}$.

104 Ibid.

105 Henderson v. Henderson [1843] 3 Hare 100, Sir James Wigram, S. 115: „[The Court] will not (except under special circumstances) permit the same parties to open the same subject of litigation in respect of a matter which might have been brought forward as part of the subject in contest [...]. The plea of res judicata applies, except in special cases, not only to points upon which the Court was actually required by the parties to form an opinion and pronounce a judgment, but to every point which properly belonged to the subject of litigation [...]."

106 Davis Kennedy v. Dandrick [1943] 2 All 606.

107 New Brunswick Railway Co. v. British and French Trust Corporation Ltd. [1939] A.C. 1; Carl Zeiss Stiftung v. Rayner \& Keeler (No 2) [1967] 1 A.C. 853.

108 Zudem gilt der Grundsatz res noviter veniens ad notitiam im schottischen, nicht aber im englischen Recht, siehe Lord Keith of Hinkel in Arnold and others v. national Westminster Bank Plc. [1991] AC 93 (HL), S. 104. Zu den darüber hinaus in der Rechtsprechung diskutieren Situationen, etwa die Nichteinbeziehung von Tatsachen in das erste Verfahren trotz reasonable diligence, siehe ders., S. $108 \mathrm{f}$.

109 Barber v. Staffordshire County Council [1996] 2 All ER 748.

110 Siehe LJ Neill in Barber v. Staffordshire County Council [1996] 2 All ER 748, S. 754. 
Gerichtsentscheidung könnte mithilfe der Restitutionsklage gemäß $§ 580$ ZPO durchbrochen werden, wenn ein entsprechender Restitutionsgrund vorliegt. Nach dem Wortlaut des $\S 580$ ZPO stellt Unionsrechtswidrigkeit keinen Grund dar. Nach § 580 Nr. 8 ZPO liegt ein Wiederaufnahmegrund vor, wenn der EGMR einen EMRK-Verstoß feststellt und das Urteil auf dieser Verletzung beruht. Ein vom EuGH festgestellter Unionsrechtsverstoß könnte durch eine analoge Anwendung dieser Regelung berücksichtigt werden. Dafür spricht, dass dies in einigen Fällen die einzige Möglichkeit ist, um einen Vertragsverstoß zu beseitigen. ${ }^{111}$ Allerdings ist das Rechtskraftprinzip auch im Unionsrecht anerkannt. Es muss daher nicht stets um jeden Preis ein Verstoß gegen das materielle Unionsrecht verhindert werden. Andererseits könnte der Prüfungsumfang des EuGH und des EGMR vergleichbar sein und so eine analoge Anwendung zulassen. Der EuGH achtet gemäß Art. 6 Abs. 3 EUV die Grundrechte, wie sie in der EMRK gewährleistet sind. Er kann daher eine mitgliedstaatliche Gerichtsentscheidung als unionsrechtswidrig erklären, wenn sie gegen die Grundrechte der EMRK verstößt, wobei er dabei die Rechtsprechung des EGMR befolgen muss. ${ }^{112}$ Dagegen spricht die unterschiedliche Ausgestaltung des Individualrechtsschutzes im Unionsrecht und dem Recht der EMRK. ${ }^{113}$ Die Individualbeschwerde zum EGMR ist erst nach rechtskräftigem Abschluss des mitgliedstaatlichen Prozesses möglich, weshalb die Restitutionsklage das notwendige Instrument der Prozessparteien ist, um ihre in der EMRK gewährten Rechte innerstaatlich umzusetzen. ${ }^{114}$ Das Unionsrecht kennt kein Direktklageverfahren der Prozessparteien. Individualrechtsschutz wird indirekt über das Vorabentscheidungsverfahren in jeder Verfahrenslage parallel zum nationalen Gerichtsverfahren ermöglicht. ${ }^{115}$ Etwas anderes könnte bei einer Verletzung der Vorlagepflicht gelten. In diesem Fall wird die effektive Durchsetzung des Unionsrechts verhindert. Die Rechtskraftdurchbrechung zur Sanktionierung einer Vorlagepflichtverletzung zu verwenden, würde jedoch das Kooperationsverhältnis zwischen dem EuGH und den nationalen Gerichten gefährden. Letztlich scheitert eine analoge Anwendung des $\S 580 \mathrm{Nr}$. 8 ZPO an einer planwidrigen Regelungslücke, da die Wiederaufnahmegründe restriktiv auszulegen sind. ${ }^{116}$

Die Rechtskraft könnte durch die Verfassungsbeschwerde gemäß Art. 93 Abs. 1 Nr. 4a GG, § 95 Abs. 2 BVerfGG durchbrochen werden. Hat diese Erfolg, wird die rechtskräftige Entscheidung aufgehoben und die Streitsache an das zuständige Gericht zurückverwiesen. Der EuGH ist ein gesetzlicher Richter im Sinne des Art. 101 Abs. 1 Satz 2 GG. Soweit ein letztinstanzliches Gericht seine Vorlagepflicht gemäß Art. 267 Abs. 3 AEUV verletzt hat, wäre das grundrechtsgleiche Recht der Prozesspartei aus Art. 101 Abs. 1 Satz 2 GG verletzt. Um nicht in die Rolle eines ,obersten Vorlagen-Kontrollgerichts" ${ }^{117}$ zu geraten, hat das Bundesverfassungsgericht die Anforderungen an eine Verletzung des Art. 101 Abs. 1

111 Meier, Zur Einwirkung des Gemeinschaftsrechts auf das nationale Verfahrensrecht im Falle höchstrichterlicher Vertragsverletzungen, EuZW 1991, S. 14.

112 EuGH, Rs. C-94/00, Roquette Frères, Slg. 2002, I-9011; Poelzig, Die Aufhebung rechtskräftiger zivilrechtlicher Urteile unter dem Einfluss des Europäischen Gemeinschaftsrechts, JZ 2007, S. 868.

113 Ibid., S. 863.

114 Ibid.

115 Ibid.

116 Germelmann, (Fn. 2), S. 287; Poelzig, (Fn. 112), S. 867.

117 Wegener, in: Calliess/Ruffert, (Fn. 17), Art. 267 AEUV, Rdnr. 35. 
Satz 2 GG erhöht. ${ }^{118}$ Nach seinen Kriterien hat eine Verfassungsbeschwerde nur Aussicht auf Erfolg, wenn die Gründe für die Vorlagepflichtverletzung bekannt sind. Die Durchbrechungsmöglichkeit ist daher auf wenige extreme Ausnahmefälle reduziert, weshalb in der Literatur eine Herabsetzung dieser Hürden gefordert wird. ${ }^{119}$

Neben diesen prozessualen Durchbrechungsmöglichkeiten bietet das materielle Recht nach $\S 826$ BGB eine Korrekturmöglichkeit. Dazu müssen drei Voraussetzungen erfüllt sein: (1.) Das Urteil muss in der Sache unrichtig sein, (2.) demjenigen, der dieses Urteil zu seinen Gunsten ausnutzt, muss die Unrichtigkeit bekannt sein und (3.) es müssen besondere Umstände vorliegen, die dieses als sittenwidrig erscheinen lassen. ${ }^{120} \mathrm{Ob}$ die Berufung auf $\S 826$ BGB die Rechtskraft beseitigt, ist umstritten. ${ }^{121}$ Der Schadensersatzanspruch beeinträchtigt jedenfalls die Durch- und Umsetzung des Urteils sowie die Wirkung der Rechtskraft. Die Möglichkeit des $\S 826$ BGB wurde von der Rechtsprechung nur für besonders extreme, eng begrenzte Ausnahmefälle geschaffen, da andernfalls die Rechtskraft ausgehöhlt, die Rechtssicherheit beeinträchtigt und der Rechtsfriede in untragbarer Weise in Frage gestellt würde. ${ }^{122}$ Die Voraussetzungen des $\S 826$ BGB dürften praktisch nicht erfüllt sein, weshalb auch auf diesem Weg die Unionsrechtswidrigkeit der Gerichtsentscheidung im deutschen Recht nicht repariert werden kann. ${ }^{123}$

An dieser Stelle wird die Bedeutung der ausgeweiteten Staatshaftung für judikatives Unrecht deutlich, wie Generalanwalt Cruz Villalón in der Rechtssache Kommission/ Slowakische Republik bereits ausgeführt hat. ${ }^{124}$ Kann die Bundesrepublik dem Gebot der effektiven Durchsetzung des Unionsrechts wegen der entgegenstehenden Rechtskraft nicht nachkommen, können zumindest die Folgen des unionsrechtswidrigen Urteils beseitigt werden. Dies wäre etwa im Bereich des Steuerrechts wie in der Rechtssache Olimpiclub nicht untragbar, da Steueransprüche und Steuererstattungen immer in Geld bestehen. ${ }^{125}$ Die Differenzierung zwischen Urteilskorrektur und Schadensersatz ist zumindest bezüglich des Ergebnisses unerheblich.

Unter Rechtsschutzgesichtspunkten ist es für die Betroffenen aber nachteilig, auf den Haftungsprozess verwiesen zu werden. Ihnen werden dann zwei langwierige Gerichtsverfahren zugemutet. Hinzu kommt, dass obgleich der Gerichtshof die Kriterien für die Staatshaftung vorgibt, die Anwendung den nationalen Gerichten obliegt. ${ }^{126}$ Dabei kann die Haftung des Staates auch unter weniger strengen Voraussetzungen ausgelöst werden, als dies unter den unionsrechtlichen Voraussetzungen der Fall wäre. ${ }^{127}$ Problematischer ist der

118 BVerfGE 82, 159, Rdnr. 144; 125, 186, Rdnr. 90 - Mangold; 128, 157, Rdnr. 103.

119 Kremer, (Fn. 12), S. 491; Wegener, (Fn. 117), Fn. 141 m.w.N.

120 BGHZ 40, 133, Rdnr. 11 ff.; 50, 119, Rdnr. 10; NJW 1998, 2818, Rdnr. 12 ff.; Rennert, in: Eyermann/Fröhler, VwGO, 13. Aufl. 2010, § 121, Rdnr. 54.

121 Rosenberg/Schwab/Gottwald, (Fn. 96), § 162, Rdnr. 4 ff.

122 BGHZ 164, 87, Rdnr. 14 ff.; Sprau, in: Palandt, Bürgerliches Gesetzbuch, 72. Aufl. 2013, § 826, Rdnr. 52.

123 Kremer, (Fn. 12), S. 486; Schmahl/Köber, (Fn. 64), S. 932.

124 Schlussanträge GA Cruz Villalón zu EuGH, Rs. C-507/08, Kommission/Slowakische Republik, Slg. 2010, I-13489, Nr. 47.

125 Vgl. de Weerth, Rückwirkende EuGH-Urteile und Bestandskraft von Steuerbescheiden, DStR 2008, S. 1671.

126 EuGH, Rs. C-302/97, Konle, Slg. 1999, I-3099, Rdnr. 58.

127 EuGH, Rs. C-446/04, Test Claimants in the FII Group Litigation, Slg. 2006, I-11753, Rdnr. 209. 
umgekehrte Fall, dass nationale Haftungsbeschränkungen die Haftung zu sehr reduzieren, wie etwa in der Rechtssache Traghetti. Ein italienisches Gesetz hatte die Staatshaftung auf Fälle von Vorsatz und grober Fahrlässigkeit beschränkt. Der Gerichtshof sah dadurch die Wirksamkeit der unionsrechtlichen Bestimmungen zum Schutz der Rechte des Einzelnen gefährdet: Der Einzelne hätte keinen gerichtlichen Schutz gegen offensichtlich unionsrechtswidrige Urteile, soweit das italienische Gesetz die Haftung des Richters ausschlösse. ${ }^{128}$ Die italienische Haftungsprivilegierung war daher zu restriktiv. Die nationale Verfahrensautonomie wird dadurch berücksichtigt, dass die nationalen Vorschriften die Natur und den Grad des Verstoßes festlegen. Einen Vorbehalt stellt der EuGH dahingehend auf, dass keine strengeren Anforderungen aufgestellt werden dürfen, als sich aus der Voraussetzung eines offenkundigen Verstoßes ergibt. ${ }^{129}$ Es ist aber gerade zweifelhaft, was der EuGH unter Offenkundigkeit versteht. ${ }^{130}$ Im englischen Recht beschränkt Section 2(5) Crown Proceedings Act 1947 die Haftung für richterliche Fehlurteile. ${ }^{131}$ Wie diese Vorschrift im Verhältnis zu den unionsrechtlichen Haftungsvorgaben steht, wurde in der Literatur bisher wenig thematisiert. ${ }^{132} \mathrm{Im}$ deutschen Recht müsste der Betroffene zunächst versuchen, durch außerordentliche Rechtsbehelfe das unionsrechtswidrige Urteil abzuändern, da ihm andernfalls gemäß $§ 839$ Abs. 3 BGB kein Schadensersatzanspruch zu Verfügung stünde. ${ }^{133}$ Die deutsche Rechtskraftdogmatik zieht die Grenzen für eine Abänderung eines rechtskräftigen Urteils recht eng. Die Verfassungsbeschwerde ist der einzige Weg die Rechtskraft zu durchbrechen, soweit ein letztinstanzliches Gericht seine Vorlagepflicht verletzt hat und die Anforderungen des Bundesverfassungsgerichts erfüllt sind. Dies dürfte jedoch nur selten der Fall sein. ${ }^{134}$ Im Haftungsprozess setzt $\S 839$ Abs. 2 BGB eine Straftat des Richters voraus. Nach Ansicht der herrschenden Literatur steht dieses Haftungsprivileg der Wirksamkeit des Unionsrechts entgegen. ${ }^{135}$ Das Unionsrecht fordert von den Mitgliedstaaten nicht, dass sie zusätzliche Primärrechtsbehelfe zur Korrektur einer Fehlentscheidung schaffen. Es muss aber ein Sekundärrechtsschutz zur Verfügung stehen, durch den der Schaden kompensiert werden kann. Soweit diese Vorschriften zur Haftungsbeschränkung der Wirksamkeit des Unionsrechts entgegenstehen, müssen sie unionsrechtskonform ausgelegt werden oder unangewendet bleiben. ${ }^{136}$ Die Umsetzung der uni-

128 EuGH, Rs. C-173/03, Traghetti/Italienische Republik, Slg. 2006, I-5177, Rdnr. 25 ff.

129 Ibid., Rdnr. 44. Die stellungnehmenden Regierungen befürworteten dagegen die nationalen Haftungsbeschränkungen (Rdnr. 28 f.).

130 Wattel, (Fn. 25), S. 178 f.; Nassimpian, (Fn. 25) S. 826.

131 Dieser lautet: No proceedings shall lie against the Crown by virtue of this section in respect of anything done or omitted to be done by any person while discharging or purporting to discharge any responsibilities of a judicial nature vested in him, or any responsibilities which he has in connection with the execution of judicial process.

132 Davies, State liability for judicial decisions in European Union and international law, International \& Comparative Law Quaterly 2012, S. 603, Fn. 62.

133 Der BGH hält $\S 839$ Abs. 3 BGB auf den unionsrechtlichen Staatshaftungsanspruch für anwendbar, siehe BGHZ 156, 294 (298).

134 Andere Ansicht Gundel, (Fn. 39), S. 15.

135 Frenz/Götzkes, (Fn. 44), S. 641; Streinz, Europarecht - Staatshaftungsrecht, JuS 2007, S. 71; Tietjen, Die Bedeutung der deutschen Richterprivilegien im System des gemeinschaftsrechtlichen Staatshaftungsrechts - Das EuGH-Urteil „Traghetti del Mediterraneo“, EWS 2007, S. 16 f. m.w.N.

136 Galetta, (Fn. 18), S. 185; Frenz/Götzkes, (Fn. 44), S. 641; Tietjen, (Fn. 135), S. 16. 
onsrechtlichen Vorgaben zur Staatshaftung für judikatives Unrecht erweist sich aufgrund der nationalen Verfahrensregelungen, aber auch des unklaren Erfordernisses eines offenkundigen Unionsrechtsverstoßes als schwierig.

\section{F. Fazit}

Die Rechtsprechungsanalyse der einschlägigen EuGH-Urteile zur Rechtskraftdurchbrechung zeigt ein zwiespältiges Bild: Zum einen betont der Gerichtshof die Bedeutung der Rechtskraft in den nationalen Rechtsordnungen und der Unionsrechtordnung, zugleich verdeutlichen die Entscheidungen aber auch, dass die Rechtskraft nationaler Gerichtsentscheidungen nicht absolut geschützt ist. Im Falle einer indirekten Kollision zwischen dem mitgliedstaatlichen Rechtskraftprinzip und dem materiellen Unionsrecht kann das unionsrechtliche Effektivitätsgebot einen Eingriff in die Verfahrensautonomie rechtfertigen. Liegt eine direkte Kollision mit dem Unionsrecht vor, genießt dieses Vorrang vor den nationalen Vorschriften, weshalb die entgegenstehende Rechtskraft bzw. deren Bindungswirkung zu durchbrechen ist. Nach dem englischen Recht wäre dies über die Konstruktion der special circumstances and special cases möglich. Im deutschen Recht kann einer Rechtskraftdurchbrechung nur schwer nachgekommen werden, da die deutsche Rechtskraftdogmatik die Grenzen für eine Abänderung eines rechtskräftigen Urteils recht eng zieht. Die Verfassungsbeschwerde ist der einzige Weg, die Rechtskraft zu durchbrechen, soweit ein letztinstanzliches Gericht seine Vorlagepflicht gemäß Art. 267 Abs. 3 AEUV verletzt hat und die Anforderungen des Bundesverfassungsgerichts erfüllt sind. Kann die Rechtskraft nicht durchbrochen werden, muss über die Staatshaftung Sekundärrechtsschutz gewährt werden. Generalanwalt Cruz Villalón schlug a posteriori in der Rechtssache Elchinov allerdings vor, zugunsten der Verfahrensautonomie eine nationale Vorschrift anzuwenden. Das dann möglicherweise unionsrechtswidrige Urteil könne mithilfe einer Schadensersatzklage angegriffen werden. ${ }^{137}$ Dem folgte der Gerichtshof nicht. ${ }^{138}$ Obgleich für den EuGH andere Aspekte maßgeblich waren, ${ }^{139}$ ist diese Entscheidung unter den Aspekten der Effektivität des Unionsrechts und des Rechtsschutzes zu begrüßen. Die Staatshaftung für judikatives Unrecht ist unter den gegenwärtigen Kriterien nur in Ausnahmefällen erfolgreich, weshalb sie für die Betroffenen nur eine zweite Chance darstellen kann, um den unionsrechtswidrigen Zustand zu beseitigen.

137 Schlussanträge GA Cruz Villalón zu EuGH, Rs. C-173/09, Elchinov, Slg. 2010, I-8889, Nr. 30. Die bulgarische Vorschrift betraf die Bindung eines untergeordneten Gerichts an die rechtliche Beurteilung des obersten Gerichts bei der Rückverweisung eines Verfahrens. Der Generalanwalt ging davon aus, dass das höchstgerichtliche Urteil materiell, aber nicht formell rechtskräftig war bzw. zumindest die Stabilität eines rechtskräftigen Urteils habe (vgl. Nr. 34). Diese Begründung ist ungenau und irreführend, da das untere Gericht das Urteil in der Sache fällt und andernfalls ein Gericht letzter Instanz i.S.d. Art. 267 Abs. 3 AEUV wäre. Daher ist das Rechtskraftprinzip nicht einschlägig, vgl. Murphy, An effective right to cross-border healthcare? - On patients, primacy and procedural autonomy: comment on Elchinov, European Law Review 2011, S. 548.

138 EuGH, Rs. C-173/09, Elchinov, Slg. 2010, I-8889, Rdnr. 32; ebenso OVG Hamburg, Beschl. v. 19.5.2011, 4 Bf 88/10, Rdnr. 28.

139 EuGH, Rs. C-173/09, Elchinov, Slg. 2010, I-8889, Rdnr. 26 ff.; dem EuGH ging es um die Vorlagemöglichkeit der nationalen Gerichte nach Art. 267 AEUV. 
Die unionsrechtskonforme Auslegung des nationalen Rechts könnte angesichts dieser Umsetzungsprobleme der unionsrechtlichen Rechtskraftdurchbrechung zu befriedigenderen Ergebnissen für die von der Gerichtsentscheidung Betroffenen führen. Sie ist geeignet, potentielle Konflikte des nationalen Verfahrensrechts mit der Effektivität des materiellen Unionsrechts schon von Anfang an zu vermeiden. ${ }^{140}$ Die damit einhergehende Funktionalisierung des mitgliedstaatlichen Verfahrensrechts stellt somit eine ausgewogene Lösung dar. ${ }^{141}$

140 Germelmann, Die Rechtskraft zwischen europäischer Mindesteffektivität und italienischem Verfassungsrecht, EuR 2010, S. 553; Galetta, (Fn. 18), S. 183 ff., welche z.B. die unionsrechtskonforme Auslegung der italienischen Staatshaftungsvorschrift für möglich hält, wodurch die EuGH-Entscheidung in Traghetti hätte vermieden werden können.

141 Galetta, (Fn. 14), S. 43; dies., (Fn. 19), S. 123. 